\title{
Leader-Follower Consensus Control of Lipschitz Nonlinear Systems by Output Feedback
}

\author{
Ahmad Sadhiqin Mohd Isira ${ }^{a}, Z_{\text {Zongyu Zuo }}^{b *}$ and Zhengtao Ding ${ }^{a}$ \\ ${ }^{a}$ Control Systems Centre, School of Electrical and Electronic Engineering, University of Manchester, \\ Sackville Street Building, Manchester M13 9PL, UK; \\ ${ }^{b}$ Seventh Research Division, and Science and Technology on Aircraft Control Laboratory, Beihang \\ University, Beijing 100191, China.
}

(Received 00 Month 20XX; final version received 00 Month 20XX)

\begin{abstract}
This paper deals with the leader-follower consensus problem of Lipschitz nonlinear systems under fixed directed communication networks. Both state and output feedback control are proposed based on state and output measurements of neighbouring agents, respectively. Laplacian matrix features are explored for the stability analysis, and the sufficient conditions are derived to solve the consensus problem. Finally, simulation results are included to demonstrate the effectiveness of the output-based consensus controller.
\end{abstract}

Keywords: Consensus, Output feedback, Multi-agent systems, Nonlinear system.

\section{Introduction}

Consensus control, as a type of cooperative control, has received considerable attention in the recent decade. Network consensus means that the states or outputs of all agents subject to a special communication network topology converge to certain quantities of interest. Hence, the consensus control design usually explores the communication structure (i.e. Laplacian matrix) to enable each dynamical subsystem in networks with a fixed or switching information connection to achieve the identical objectives. Many applications, such as formation control, synchronization and others, are based on the consensus algorithms.

In the modern context of multi-agent systems, numerous results have been obtained for the consensus problem (Fax et al. 2004; Olfati-Saber et al. 2004, 2003). Information consensus for first-order (single-integrator) systems has been investigated in the papers such as (Ren 2007; Cao et al. 2010; Chen et al. 2011; Ren 2007), to name a few. It is then expanded to second order (double-integrator) consensus problem as shown in (Ren et al. 2007) and higher order consensus in (Ren et al. 2007; He et al. 2011). Some are analysed based on leader-follower configuration for single leader (Cai et al. 2014; Hong et al. 2010; Cheng et al. 2010) and multi-leader configuration (Wang et al. 2014). Consensus for complex systems has also been studied as in (Zhang et al. 2011; Ni et al. 2010), including the study of consensus problem for the systems with nonlinear terms ( $\mathrm{Li}$ et al. 2010; Chen et. al. 2009; Song et al. 2010), with delay ( $\mathrm{Li}$ et al. 2011; Liu et al. 2012; Cui et al. 2012) or the linear systems with nonlinear consensus protocols (Zuo et al. 2014; Su et al. 2013). Although some results on consensus control with nonlinearity have been stated in (Liu et al. 2013; Ding 2013; Dong et al. 2014; Li et al. 2012), most are restricted to

*Corresponding author: Zongyu Zuo. E-mail: zzybobby@buaa.edu.cn. 
local stability, or to certain connections of the network such as the existence of a tree structure in the connection, or certain types of nonlinearities. There are a few papers that deal with consensus problem of high-order dynamic systems with Lipschitz nonlinearities. A result on the consensus for Lipchitz nonlinear systems derived in ( $\mathrm{Li}$ et al. 2012) assumed that the left eigenvector corresponding to the eigenvalue of the Laplacian matrix at zero has no zero elements. Ding (Ding 2014) investigated the global consensus control for a network-connected system in the presence of a Lipschitz nonlinearity with a fairly general assumption on the connection structure.

In real-life applications, it is often impossible to obtain the measurements of all system states. In such a case, state feedback controller clearly are not applicable, but the observer-based controller, or output feedback controller can provide a viable solution. There have been a number of results developed on the observer design for Lipschitz nonlinear systems (Rajamani 1998; Rajamani et al. 2002; Aboky et al. 2002; Phanomchoeng et al. 2010; Ding 2013).

Since few results on the output feedback consensus control for Lipchitz nonlinear multi-agent systems have been reported, this paper presents a new consensus controller for such systems by output feedback. The basic structure of the controller is built upon the works of Li et.al ( $\mathrm{Li}$ et al. 2010, 2009), and Ding (Ding 2014). However, apart from the leader-follower configuration, the key feature different from the works mentioned includes two aspects: (i) a Lipschitz nonlinearity in the subsystem dynamics is considered, and (ii) a consensus controller based on the outputs of neighbouring subsystems is proposed. The Lyapunov tools are employed for the consensus stability analysis and several sufficient conditions are derived from careful study of the connection topology (i.e. the Laplacian structure). Finally, simulations are given to validate the proposed consensus controller.

The rest of this paper is organized as follows. Section 2 presents the problem and some basic notations on graph theory. The leader-follower consensus with state feedback control design and the consensus observer design are given respectively in Section 3 and Section 4. Section 5 presents the main result on the leader-following consensus by output feedback. Simulation results are included in 6. Section 7 concludes this paper.

Notations: Throughout the paper, $\mathbb{R}$ denotes the set of real numbers. We use the 2-norm (i.e., Euclidean norm) to measure the size of a vector $x \in \mathbb{R}^{n}$, defined by $\|x\|=\sqrt{x^{T} x}$, and use the induced norm corresponding to the 2 -norm for vectors to measure the size of a matrix $A \in \mathbb{R}^{n \times n}$, defined by $\|A\|=\sup _{x \neq 0} \frac{\|A x\|}{\|x\|}$. The symbol $\otimes$ denotes the Kronecker product of matrices (Horn 1990).

\section{Problem Statement}

Consider $N+1$ nonlinear subsystems with identical dynamics, described as

$$
\begin{aligned}
\dot{x}_{i} & =A x_{i}+\phi\left(x_{i}\right)+B u_{i} \\
y_{i} & =C x_{i}
\end{aligned}
$$

where for $i=0, \ldots, N, x_{i} \in \mathbb{R}^{n}$ is the state vector of the subsystem, $u_{i} \in \mathbb{R}^{p}$ is the input of the $i$ th subsystem, and $y_{i} \in \mathbb{R}^{q}$ is the measured output vector, $A \in \mathbb{R}^{n \times n}, B \in \mathbb{R}^{n \times p}$, and $C \in \mathbb{R}^{q \times n}$ are appropriate matrices, and $\phi: \mathbb{R}^{n} \rightarrow \mathbb{R}^{n}$ is a Lipschitz nonlinear function with the Lipschitz constant $\gamma$, i.e., for any two constant vectors $a, b \in \mathbb{R}^{n}$, we have

$$
\|\phi(a)-\phi(b)\| \leq \gamma\|a-b\|
$$

Without loss of generality, a subsystem indexed by 0 is assigned as the leader with $u_{0}=0$ and the subsystems indexed by $i=1, \ldots, N$ are referred to as followers.

The connections between the subsystems are specified by a directed graph $\mathcal{G}$ which consists of a set of vertices denoted by $\mathcal{V}$ and a set of edges denoted by $\mathcal{E}$. A vertex represents a subsystem 
and each edge represents a connection. The adjacency matrix $Q$ associated with the graph $\mathcal{G}$ is defined with elements $q_{i j}=1$ if there is a connection between subsystem $j$ to $i$, and $q_{i j}=0$ otherwise. The Laplacian matrix $\mathcal{L}=\left\{l_{i j}\right\}$ is commonly defined as

$$
\begin{aligned}
& l_{i j}=-q_{i j}, \text { if } j \neq i \\
& l_{i i}=\sum_{j=1, j \neq i}^{N} q_{i j}
\end{aligned}
$$

A couple of assumptions are needed

Assumption 1. Triple $(A, B, C)$ is controllable and observable.

Assumption 2. The communication network $\mathcal{G}$ of the multi-agent systems contains a directed spanning tree (Godsil et al. (2001)) with the leader as the root.

Since the leader has no neighbours, $\mathcal{L}$ can be partitioned as

$$
\mathcal{L}=\left[\begin{array}{cc}
0 & 0_{1 \times N} \\
l_{1} & \overline{\mathcal{L}}
\end{array}\right]
$$

where $\overline{\mathcal{L}} \in \mathbb{R}^{N \times N}$ and $l_{1}=\left[l_{10}, \ldots, l_{N 0}\right]^{T} \in \mathbb{R}^{N \times 1}$.

Lemma 1 ((Hong et al. 2006)). If the Assumption 2 holds, then all the eigenvalues of $\overline{\mathcal{L}}$ have positive real parts.

The control objective is to design the leader-follower consensus controller using measured output information of the neighbouring agents such that the states of the follower subsystems in a group asymptotically track the state of the leader subsystem.

\section{Leader-Follower State Feedback Consensus Controller}

The controller proposed using the relative state information takes the structure

$$
u_{i}=K \sum_{j=0}^{N} l_{i j} x_{j}=K \sum_{j=1}^{N} l_{i j}\left(x_{j}-x_{0}\right)
$$

where $K \in \mathbb{R}^{p \times n}$ is a constant control gain matrix to be designed later and the fact $l_{i 0}=$ $-\sum_{j=1}^{N} l_{i j}$ is used. The leader-follower consensus problem is said to be solved if

$$
x_{i}(t) \rightarrow x_{0}(t), \forall i=1, \ldots, N \text { as } t \rightarrow \infty
$$

We first introduce the disagreement between the leader and follower subsystem's state denoted by

$$
\xi_{i}=x_{i}-x_{0}
$$

With $\xi=\left[\xi_{1}^{T}, \ldots, \xi_{N}^{T}\right]^{T}, \Phi(x)=\left[\phi^{T}\left(x_{1}\right), \ldots, \phi^{T}\left(x_{N}\right)\right]^{T}$ and $\Phi\left(x_{0}\right)=\mathbf{1} \otimes \phi\left(x_{0}\right)$, the compact form of closed-loop dynamics of $\xi$ are given by

$$
\dot{\xi}=\left(I_{N} \otimes A-\overline{\mathcal{L}} \otimes B K\right) \xi+\Phi(x)-\Phi\left(x_{0}\right)
$$


where $\overline{\mathcal{L}}$ is defined in (6).

Let us introduce nonsingular matrices $T \in \mathbb{R}^{N \times N}$ and $T^{-1} \in \mathbb{R}^{N \times N}$ such that

$$
T^{-1} \overline{\mathcal{L}} T=\bar{J}
$$

with $\bar{J}$ being a block-diagonal matrix of real Jordan form

$$
\left[\begin{array}{ccccccc}
\bar{J}_{1} & & & & & \\
& \bar{J}_{2} & & & & \\
& & & & & & \\
& & & \bar{J}_{p} & & & \\
& & & \bar{J}_{p+1} & & \\
& & & & & \ddots & \\
& & & & & \bar{J}_{q}
\end{array}\right]
$$

where $\bar{J}_{k} \in \mathbb{R}^{n_{k}}$ for $k=1, \ldots, p$ are the Jordan blocks for real eigenvalues $\bar{\lambda}_{k}>0$ with the multiplicity $n_{k}$ in the form

$$
\bar{J}_{k}=\left[\begin{array}{cccccc}
\bar{\lambda}_{k} & 1 & & & \\
& \bar{\lambda}_{k} & 1 & & \\
& & \ddots & \ddots & \\
& & & \bar{\lambda}_{k} & 1 \\
& & & & \bar{\lambda}_{k}
\end{array}\right]
$$

and $\bar{J}_{k} \in \mathbb{R}^{2 n_{k}}$ for $k=p+1, \ldots, q$ are the Jordan blocks for conjugate eigenvalues $\bar{\alpha}_{k} \pm \mathrm{j} \bar{\beta}_{k}$, $\bar{\alpha}_{k}>0$ and $\bar{\beta}_{k}>0$ with multiplicity $n_{k}$ in the form

$$
\bar{J}_{k}=\left[\begin{array}{ccccc}
\mu\left(\bar{\alpha}_{k}, \bar{\beta}_{k}\right) & I_{2} & & & \\
& \mu\left(\bar{\alpha}_{k}, \bar{\beta}_{k}\right) & I_{2} & & \\
& & \ddots & \ddots & \\
& & & \mu\left(\bar{\alpha}_{k}, \bar{\beta}_{k}\right) & I_{2} \\
& & & & \mu\left(\bar{\alpha}_{k}, \bar{\beta}_{k}\right)
\end{array}\right]
$$

with $I_{2}$ the identity matrix in $\mathbb{R}^{2 \times 2}$ and

$$
\mu\left(\bar{\alpha}_{k}, \bar{\beta}_{k}\right)=\left[\begin{array}{cc}
\bar{\alpha}_{k} & \bar{\beta}_{k} \\
-\bar{\beta}_{k} & \bar{\alpha}_{k}
\end{array}\right] \in \mathbb{R}^{2 \times 2}
$$

To exploit the structure of $\overline{\mathcal{L}}$, two other transformations are introduced

$$
\begin{gathered}
\eta=\left(T^{-1} \otimes I_{n}\right) \xi \\
\Psi\left(x, x_{0}\right)=\left(T^{-1} \otimes I_{n}\right)\left(\Phi(x)-\Phi\left(x_{0}\right)\right)
\end{gathered}
$$

and thus we obtain the transformed closed-loop network dynamics as

$$
\dot{\eta}=\left(I_{N} \otimes A-\bar{J} \otimes B K\right) \eta+\Psi\left(x, x_{0}\right)
$$

where $\eta=\left[\eta_{1}^{T}, \ldots, \eta_{N}^{T}\right]^{T}, \Psi\left(x, x_{0}\right)=\left[\psi_{1}^{T}\left(x, x_{0}\right), \ldots, \psi_{N}^{T}\left(x, x_{0}\right)\right]^{T}$ and $\bar{J}$ takes the Jordan matrix form of $(12)$. 


\subsection{Stability Analysis}

For the stability analysis, we need to establish a bound of the nonlinear function in terms of the transformed $\eta$.

Lemma 2. For nonlinear element $\psi_{i}$ of the nonlinear term $\Psi$ in the transformed closed-loop network dynamics (16), a bound can be established in terms of the state $\eta$ as shown by

$$
\left\|\psi_{i}\right\| \leq \frac{\gamma_{0}}{\sqrt{N}}\|\eta\|
$$

with

$$
\gamma_{0}=\gamma \lambda_{\sigma}\left(T^{-1}\right) \lambda_{\sigma}(T) \sqrt{N}
$$

where $\lambda_{\sigma}(\cdot)$ denotes the maximum singular value of a matrix.

Proof. The approach of the derivation of (17) and (18) is similar to Lemma 3.2 in (Ding 2014). From (15) we have

$$
\begin{aligned}
\left\|\psi_{i}\left(x, x_{0}\right)\right\| & \leq\left\|t_{i} \otimes I_{n}\right\|\left\|\Phi(x)-\Phi\left(x_{0}\right)\right\| \\
& \leq\left\|T^{-1} \otimes I_{n}\right\| \gamma\left\|x-x_{0}\right\| \\
& \leq \lambda_{\sigma}\left(T^{-1}\right) \lambda_{\sigma}\left(I_{n}\right) \gamma\left\|x-x_{0}\right\| \\
& =\lambda_{\sigma}\left(T^{-1}\right) \gamma\|\xi\|
\end{aligned}
$$

where $t_{i}$ denotes the $i^{t h}$ row of $T^{-1}$ and $\lambda_{\sigma}\left(I_{n}\right)=1$ has been used. And from (14) we have

$$
\begin{aligned}
\|\xi\| & \leq\left\|T \otimes I_{n}\right\|\|\eta\| \\
& \leq \lambda_{\sigma}(T)\|\eta\|
\end{aligned}
$$

Then, it is easy to get

$$
\begin{aligned}
\left\|\psi_{i}\left(x, x_{0}\right)\right\| & \leq \frac{\gamma \lambda_{\sigma}\left(T^{-1}\right) \lambda_{\sigma}(T) \sqrt{N}}{\sqrt{N}}\|\eta\| \\
& =\frac{\gamma_{0}}{\sqrt{N}}\|\eta\|
\end{aligned}
$$

with $\gamma_{0}$ as in (18).

The bound derived in the lemma above will be used for the control gain design in the following theorem.

Theorem 1. For a network-connected nonlinear systems (1) with the communication topology $\mathcal{G}$ satisfying Assumption 2, the consensus control design (7) with $K=B^{T} P$ solves the leaderfollower consensus problem if there exists a solution of $P=P^{T}>0$ specified in one of the following two cases:

(1) If the eigenvalues of the matrix $\overline{\mathcal{L}}$ are distinct, i.e., $n_{k}=1$ for $k=1, \ldots, q$, the matrix $P$ satisfies

$$
A^{T} P+P A-2 \bar{\alpha} P B B^{T} P+\kappa P P+\frac{\gamma_{0}^{2}}{\kappa} I_{n}<0
$$


with $\kappa$ being any positive real number and $\bar{\alpha}=\min \left\{\bar{\lambda}_{1}, \ldots, \bar{\lambda}_{p}, \bar{\alpha}_{p+1}, \ldots, \bar{\alpha}_{q}\right\}$.

(2) If the matrix $\overline{\mathcal{L}}$ have multiple eigenvalues, i.e., $n_{k}>1$ for any $k \in\{1, \ldots, q\}$, the matrix $P$ satisfies

$$
A^{T} P+P A-2(\bar{\alpha}-1) P B B^{T} P+\kappa P P+\frac{\gamma_{0}^{2}}{\kappa} I_{n}<0
$$

with $\kappa$ being any positive real number.

Proof. Within each real Jordan block $\bar{J}_{k}$, for $k \leq p$ we have, for $i=N_{k-1}+1, \ldots, N_{k}-1$,

$$
\dot{\eta}_{i}=\left(A-\bar{\lambda}_{i} B K\right) \eta_{i}-B K \eta_{i+1}+\psi_{i}(x)
$$

and

$$
\dot{\eta}_{i}=\left(A-\bar{\lambda}_{i} B K\right) \eta_{i}+\psi_{i}(x)
$$

for $i=N_{k}$.

For the state variable associated with the Jordan blocks $J_{k}$ for $k>p$ corresponding to complex eigenvalues, we consider the dynamics of the state variables in pairs. For notational convenience, let

$$
\begin{aligned}
& i_{1}(j)=N_{k-1}+2 j-1 \\
& i_{2}(j)=N_{k-1}+2 j
\end{aligned}
$$

for $j=1, \ldots, n_{k} / 2$. The dynamics of $\eta_{i_{1}}$ and $\eta_{i_{2}}$ for $j=1, \ldots, n_{k} / 2-1$ are expressed by

$$
\begin{aligned}
& \dot{\eta}_{i_{1}}=\left(A-\bar{\alpha}_{k} B K\right) \eta_{i_{1}}-\beta_{k} B K \eta_{i_{2}}-B K \eta_{i_{1}+2}+\psi_{i_{1}}(x) \\
& \dot{\eta}_{i_{2}}=\left(A-\bar{\alpha}_{k} B K\right) \eta_{i_{2}}+\beta_{k} B K \eta_{i_{1}}-B K \eta_{i_{2}+2}+\psi_{i_{2}}(x)
\end{aligned}
$$

and

$$
\begin{aligned}
& \dot{\eta}_{i_{1}}=\left(A-\bar{\alpha}_{k} B K\right) \eta_{i_{1}}-\beta_{k} B K \eta_{i_{2}}+\psi_{i_{1}}(x) \\
& \dot{\eta}_{i_{2}}=\left(A-\bar{\alpha}_{k} B K\right) \eta_{i_{2}}+\beta_{k} B K \eta_{i_{1}}+\psi_{i_{2}}(x)
\end{aligned}
$$

for $j=n_{k} / 2$.

Let $W_{i}=\eta_{i}^{T} P \eta_{i}$. Choose $V_{k}=\sum_{j=1}^{n_{k}} \sigma^{2(j-1)} W_{j+N_{k-1}}$ for $k=1, \ldots, p$ and $V_{k}=$ $\sum_{j=1}^{n_{k} / 2} \sigma^{2(j-1)}\left(W_{i_{1}(j)}+W_{i_{2}(j)}\right)$ for $k=p+1, \ldots, q$, where $\sigma>0$. Then we consider the Lyapunov function $V=\sum_{i=1}^{q} V_{k}$. With $K=B^{T} P$, we have the following results.

Case 1. For the distinct eigenvalues, we can obtain that

$$
\dot{V} \leq \sum_{i=1}^{N} \eta_{i}^{T}\left(A^{T} P+P A-2 \bar{\alpha} P B B^{T} P+\kappa P P+\frac{\gamma_{0}^{2}}{\kappa} I_{n}\right) \eta_{i}
$$

The condition (19) guarantees $\dot{V}<0$. 
Case 2. For multiple eigenvalues, we can obtain that

$$
\begin{aligned}
\dot{V} & \leq \sum_{i=1}^{N} \eta_{i}^{T}\left[A^{T} P+P A-2\left(\bar{\alpha}-\frac{1}{\sigma}\right) P B B^{T} P+\kappa P P+\frac{\gamma_{0}^{2}}{\kappa} I_{n}\right] \eta_{i} . \\
& \leq \sum_{i=1}^{N} \eta_{i}^{T}\left[A^{T} P+P A-2(\bar{\alpha}-1) P B B^{T} P+\kappa P P+\frac{\gamma_{0}^{2}}{\kappa} I_{n}\right] \eta_{i}
\end{aligned}
$$

with $\sigma=1$. The condition (20) guarantees $\dot{V}<0$.

Hence we conclude that $\eta_{i}(t) \rightarrow 0, \forall i=1, \ldots, N$ as $t \rightarrow \infty$. This completes the proof.

Some of the derivations of the proof have been omitted to save the space. We refer readers to (Ding 2014) for similar information in detail.

\section{Consensus Observer}

The consensus observer for each subsystem is proposed as

$$
\dot{\hat{x}}_{i}=A \hat{x}_{i}+B u_{i}+\phi\left(\hat{x}_{i}\right)+L \sum_{j=1}^{N} l_{i j}\left(y_{j}-C \hat{x}_{j}\right)
$$

where $\hat{x}_{i} \in \mathbb{R}^{n}$ for $i=1, \ldots, N$ is the estimated state of each subsystem (follower) and $L \in \mathbb{R}^{n \times q}$ is the observer gain matrix to be determined later.

Remark 1. Differently from the traditional Luenberger observer which uses the absolute output information, the proposed consensus observer (24) only uses the relative output and estimated output information of the neighbours via network connections to produce the estimated value of the subsystem itself.

The estimation error dynamics can be then derived as

$$
\dot{\tilde{x}}_{i}=A \tilde{x}_{i}+\phi\left(x_{i}\right)-\phi\left(\hat{x}_{i}\right)-L C \sum_{j=1}^{N} l_{i j} \tilde{x}_{j}
$$

where $\tilde{x}_{i}=x_{i}-\hat{x}_{i}$. With $\tilde{x}=\left[\tilde{x}_{1}^{T}, \ldots, \tilde{x}_{N}^{T}\right]^{T}, \Phi(x)=\left[\phi^{T}\left(x_{1}\right), \ldots, \phi^{T}\left(x_{N}\right)\right]^{T}$ and $\Phi(\hat{x})=$ $\left[\phi^{T}\left(\hat{x}_{1}\right), \ldots, \phi^{T}\left(\hat{x}_{N}\right)\right]^{T}$, the estimation error dynamics (25) for each subsystem can be stacked into a compact form as

$$
\dot{\tilde{x}}=\left(I_{N} \otimes A-\overline{\mathcal{L}} \otimes L C\right) \tilde{x}+\Phi(x)-\Phi(\hat{x})
$$

where $\overline{\mathcal{L}}$ is defined in (6). Likewise, we introduce the transformation

$$
\begin{gathered}
\tilde{\eta}=\left(T^{-1} \otimes I_{n}\right) \tilde{x} \\
\bar{\Psi}(x, \hat{x})=\left(T^{-1} \otimes I_{n}\right)(\Phi(x)-\Phi(\hat{x}))
\end{gathered}
$$

with $T^{-1} \overline{\mathcal{L}} T=\bar{J}$ defined in (12). Hence, the transformed dynamics of the consensus observer 
are given by

$$
\dot{\tilde{\eta}}=\left(I_{N} \otimes A-\bar{J} \otimes L C\right) \tilde{\eta}+\bar{\Psi}(x, \hat{x})
$$

where $\tilde{\eta}=\left[\tilde{\eta}_{1}^{T}, \ldots, \tilde{\eta}_{N}^{T}\right]^{T}, \bar{\Psi}(x, \hat{x})=\left[\bar{\psi}_{1}^{T}(x, \hat{x}), \ldots, \bar{\psi}_{N}^{T}(x, \hat{x})\right]^{T}$.

Remark 2. Similar to the previous section, the estimation error dynamics (26) is now turned into a diagonally dominant matrix, which facilitates the stability analysis.

\subsection{Stability Analysis}

Similar to the previous section, the bound of $\bar{\psi}_{i}(x, \hat{x})$ in terms of $\tilde{\eta}$ is needed.

Lemma 3. For nonlinear element $\bar{\psi}_{i}$ of the nonlinear term $\bar{\Psi}$ in the transformed closed-loop network dynamics (29), a bound can be established in terms of $\tilde{\eta}$ as shown by

$$
\left\|\bar{\psi}_{i}(x, \hat{x})\right\| \leq \frac{\gamma_{0}}{\sqrt{N}}\|\tilde{\eta}\|
$$

with

$$
\gamma_{0}=\gamma \lambda_{\sigma}\left(T^{-1}\right) \lambda_{\sigma}(T) \sqrt{N}
$$

where $\lambda_{\sigma}(\cdot)$ denotes the maximum singular value of a matrix.

Proof. From (28), we have

$$
\left\|\bar{\psi}_{i}(x, \hat{x})\right\| \leq\left\|t_{i} \otimes I_{n}\right\|\|\Phi(x)-\Phi(\hat{x})\|
$$

where $t_{i}$ is the $i^{\text {th }}$ row of $T^{-1}$. And from (27), we have

$$
\|\tilde{x}\| \leq\left\|T \otimes I_{n}\right\|\|\tilde{\eta}\|
$$

Hence, similar to the proof in Lemma 2, the following can be obtained

$$
\begin{aligned}
\left\|\bar{\psi}_{i}(x, \hat{x})\right\| & \leq \lambda_{\sigma}\left(T^{-1}\right) \gamma\|\tilde{x}\| \\
& \leq \lambda_{\sigma}\left(T^{-1}\right) \gamma \lambda_{\sigma}(T)\|\tilde{\eta}\| \\
& =\frac{\gamma_{0}}{\sqrt{N}}\|\tilde{\eta}\|
\end{aligned}
$$

with $\gamma_{0}$ as in (31).

The bound obtained in the lemma above will be used for the consensus observer gain design in the following theorem.

Theorem 2. For a network-connected dynamic system (1) with communication topology $\mathcal{G}$ satisfying Assumption 2, (24) with $L=P^{-1} C^{T}$ is an asymptotically stable observer for the system (1) if there exists a solution $P=P^{T}>0$ specified in the following of the two cases:

(1) If the eigenvalues of the matrix $\overline{\mathcal{L}}$ are distinct, i.e., $n_{k}=1$ for $k=1, \ldots, q$ the matrix $P=P^{T}>0$ satisfies

$$
A^{T} P+P A-2 \bar{\alpha} C^{T} C+\kappa P P+\frac{\gamma_{0}^{2}}{\kappa} I_{n}<0
$$


with $\kappa$ being any positive real number and $\bar{\alpha}=\min \left\{\bar{\lambda}_{1}, \ldots, \bar{\lambda}_{p}, \bar{\alpha}_{p+1}, \ldots, \bar{\alpha}_{q}\right\}$.

(2) If the matrix $\overline{\mathcal{L}}$ have multiple eigenvalues, i.e., $n_{k}>1$ for any $k \in\{1, \ldots, q\}$, the matrix $P=P^{T}>0$ satisfies

$$
A^{T} P+P A-2(\bar{\alpha}-1) C^{T} C+\kappa P P+\frac{\gamma_{0}^{2}}{\kappa} I_{n}<0
$$

with $\kappa$ being any positive real number.

Proof. Similar to the proof of Theorem 1, for $k \leq p$ we have

$$
\dot{\tilde{\eta}}_{i}=\left(A-\bar{\lambda}_{i} L C\right) \tilde{\eta}_{i}-L C \tilde{\eta}_{i+1}+\bar{\psi}_{i}(x)
$$

with $i=N_{k-1}+1, \ldots, N_{k}-1$ and

$$
\dot{\tilde{\eta}}_{i}=\left(A-\bar{\lambda}_{i} L C\right) \tilde{\eta}_{i}+\bar{\psi}_{i}(x)
$$

for $i=N_{k}$.

For $k>p$, we have

$$
\begin{aligned}
& \dot{\tilde{\eta}}_{i_{1}}=\left(A-\bar{\alpha}_{k} L C\right) \tilde{\eta}_{i_{1}}-\beta_{k} L C \tilde{\eta}_{i_{2}}-L C \tilde{\eta}_{i_{1}+2}+\psi_{i_{1}}(x) \\
& \dot{\tilde{\eta}}_{i_{2}}=\left(A-\bar{\alpha}_{k} L C\right) \tilde{\eta}_{i_{2}}+\beta_{k} L C \tilde{\eta}_{i_{1}}-L C \tilde{\eta}_{i_{2}+2}+\psi_{i_{2}}(x)
\end{aligned}
$$

with $j=1, \ldots, n_{k} / 2-1$ and

$$
\begin{aligned}
& \dot{\tilde{\eta}}_{i_{1}}=\left(A-\bar{\alpha}_{k} L C\right) \tilde{\eta}_{i_{1}}-\beta_{k} L C \tilde{\eta}_{i_{2}}+\bar{\psi}_{i_{1}}(x) \\
& \dot{\tilde{\eta}}_{i_{2}}=\left(A-\bar{\alpha}_{k} L C\right) \tilde{\eta}_{i_{2}}+\beta_{k} L C \tilde{\eta}_{i_{1}}+\bar{\psi}_{i_{2}}(x)
\end{aligned}
$$

for $j=n_{k} / 2$.

Let $\bar{W}_{i}=\tilde{\eta}_{i}^{T} P \tilde{\eta}_{i}$. Choose $\bar{V}_{k}=\sum_{j=1}^{n_{k}} \sigma^{2(j-1)} \bar{W}_{j+N_{k-1}}$ for $k=1, \ldots, p$ and $\bar{V}_{k}=$ $\sum_{j=1}^{n_{k} / 2} \sigma^{2(j-1)}\left(\bar{W}_{i_{1}(j)}+\bar{W}_{i_{2}(j)}\right)$ for $k=p+1, \ldots, q$, where $\sigma>0$. Then we consider the Lyapunov function $\bar{V}=\sum_{i=1}^{q} \bar{V}_{k}$. With $\sigma=1$ and $L=P^{-1} C^{T}$, we have the following results.

Case 1. For the distinct eigenvalues, we can obtain that

$$
\dot{\bar{V}} \leq \sum_{i=1}^{N} \tilde{\eta}_{i}^{T}\left(A^{T} P+P A-2 \bar{\alpha} C^{T} C+\kappa P P+\frac{\gamma_{0}^{2}}{\kappa} I_{n}\right) \tilde{\eta}_{i}
$$

Case 2. For multiple eigenvalues, we can obtain that

$$
\dot{\bar{V}} \leq \sum_{i=1}^{N} \tilde{\eta}_{i}^{T}\left[A^{T} P+P A-2(\bar{\alpha}-1) C^{T} C+\kappa P P+\frac{\gamma_{0}^{2}}{\kappa} I_{n}\right] \tilde{\eta}_{i}
$$

Therefore, conditions (34) and (35) guarantee that $\dot{\bar{V}}<0$ for both cases. Hence we conclude that $\tilde{\eta}_{i}(t) \rightarrow 0, \forall i=1, \ldots, N$ as $t \rightarrow \infty$. This completes the proof. 


\section{Leader-follower Consensus Controller with State Estimation}

The output feedback consensus controller takes the following structure

$$
u_{i}=-K\left(\hat{x}_{i}-x_{0}\right)
$$

where $\hat{x}_{i} \in \mathbb{R}^{n}$ for $i=1, \ldots, N$ are the estimated states defined in (24) and the consensus controller gain $K \in \mathbb{R}^{p \times n}$ is to be determined later.

Remark 3. The controller utilizes the estimated information provided by the consensus observer for each of the subsystem. It is worth noting that the consensus controller in (37) is an output feedback controller, since the consensus observer in (24) only depends on the outputs of the subsystems.

Remark 4. It is worth mentioning that the implementation of the consensus controller (37) requires the state of the leader to be accessed by the followers. This requirement is due to the nonlinearity of each subsystem. In other words, it is not needed if all subsystems in a group are linear.

With (3) and (37), system (1) can be written as

$$
\dot{x}_{i}=(A-B K) x_{i}+B K x_{0}+B K \tilde{x}_{i}+\phi\left(x_{i}\right)
$$

and with (26), the augmented closed-loop network dynamics can be stacked into a compact form as

$$
\begin{aligned}
{\left[\begin{array}{c}
\dot{\xi} \\
\dot{\tilde{x}}
\end{array}\right]=} & {\left[\begin{array}{cc}
I_{N} \otimes(A-B K) & I_{N} \otimes B K \\
0 & I_{N} \otimes A-\overline{\mathcal{L}} \otimes L C
\end{array}\right]\left[\begin{array}{l}
\xi \\
\tilde{x}
\end{array}\right] } \\
& +\left[\begin{array}{c}
\Phi(x)-\Phi\left(x_{0}\right) \\
\Phi(x)-\Phi(\hat{x})
\end{array}\right]
\end{aligned}
$$

By using the same transformation (27) for $\tilde{\eta}$, we have the transformed network dynamics

$$
\begin{aligned}
{\left[\begin{array}{c}
\dot{\xi} \\
\dot{\tilde{\eta}}
\end{array}\right]=} & {\left[\begin{array}{cc}
I_{N} \otimes(A-B K) & I_{N} \otimes B K \\
0 & I_{N} \otimes A-\bar{J} \otimes L C
\end{array}\right]\left[\begin{array}{l}
\xi \\
\tilde{\eta}
\end{array}\right] } \\
& +\left[\begin{array}{c}
\Phi(x)-\Phi\left(x_{0}\right) \\
\bar{\Psi}(x, \hat{x})
\end{array}\right]
\end{aligned}
$$

We summarize the main result of this paper in the following theorem.

Theorem 3. For a network-connected dynamic system (1) with the associated Laplacian matrix that satisfies Assumptions 1-2, the observer-based controller (37) with $K=B^{T} P_{1}$, and $L=$ $P_{2}^{-1} C^{T}$ solve the leader-follower consensus problem if there exist solutions $P_{1}=P_{1}^{T}>0$ and $P_{2}=P_{2}^{T}>0$ specified in one of the following cases:

(1) If the eigenvalues of the matrix $\overline{\mathcal{L}}$ are distinct, i.e., $n_{k}=1$ for $k=1, \ldots, q$, the matrices $P_{1}$ and $P_{2}$ satisfy

$$
A^{T} P_{1}+P_{1} A-P_{1} B B^{T} P_{1}+\kappa_{1} P_{1} P_{1}+\frac{\gamma^{2}}{\kappa_{1}} I_{n}<0
$$

and

$$
A^{T} P_{2}+P_{2} A-2 \bar{\alpha} C^{T} C+\kappa_{2} P_{2} P_{2}+\frac{\gamma_{0}^{2}}{\kappa_{2}} I_{n}+P_{1} B B^{T} P_{1}<0
$$


with $\kappa_{1}$ and $\kappa_{2}$ being any positive real numbers, $\bar{\alpha}=\min \left\{\bar{\lambda}_{1}, \ldots, \bar{\lambda}_{p}, \bar{\alpha}_{p+1}, \ldots, \bar{\alpha}_{q}\right\}, \gamma$ defined in (3) and $\gamma_{0}$ defined in (31).

(2) If the eigenvalues of the matrix $\overline{\mathcal{L}}$ are multiple eigenvalues, i.e., $n_{k}>1$ for any $k \in$ $\{1, \ldots, q\}$, the matrices $P_{1}$ and $P_{2}$ satisfy

$$
A^{T} P_{1}+P_{1} A-P_{1} B B^{T} P_{1}+\kappa_{1} P_{1} P_{1}+\frac{\gamma^{2}}{\kappa_{1}} I_{n}<0
$$

and

$$
A^{T} P_{2}+P_{2} A-2(\bar{\alpha}-1) C^{T} C+\kappa_{2} P_{2} P_{2}+\frac{\gamma_{0}^{2}}{\kappa_{2}} I_{n}+P_{1} B B^{T} P_{1}<0
$$

with $\kappa_{1}$ and $\kappa_{2}$ being any positive real numbers.

Proof. Let $V_{1}=\sum_{i=1}^{N} \xi_{i}^{T} P_{1} \xi_{i}$ and taking derivative of $V_{1}$ along (40) yields

$$
\begin{aligned}
\dot{V}_{1} & =\sum_{i=1}^{N}\left[(A-B K) \xi_{i}+B K \tilde{\eta}_{i}+\phi\left(x_{i}\right)-\phi\left(x_{0}\right)\right]^{T} P_{1} \xi_{i}+\sum_{i=1}^{N} \xi_{i}^{T} P_{1}\left[(A-B K) \xi_{i}+B K \tilde{\eta}_{i}+\phi\left(x_{i}\right)-\phi\left(x_{0}\right)\right] \\
& =\sum_{i=1}^{N} \xi_{i}^{T}\left(A^{T} P_{1}+P_{1} A-2 P_{1} B K\right) \xi_{i}+2 \xi_{i}^{T} P_{1} B K \tilde{\eta}_{i}+\sum_{i=1}^{N} 2 \xi_{i}^{T} P_{1}\left(\phi\left(x_{i}\right)-\phi\left(x_{0}\right)\right) \\
& \leq \sum_{i=1}^{N} \xi_{i}^{T}\left(A^{T} P_{1}+P_{1} A-P_{1} B B^{T} P_{1}+\kappa_{1} P_{1} P_{1}\right) \xi_{i}+\sum_{i=1}^{N} \tilde{\eta}_{i}^{T} P_{1} B B^{T} P_{1} \tilde{\eta}_{i}+\sum_{i=1}^{N} \frac{1}{\kappa_{1}}\left\|\phi\left(x_{i}\right)-\phi\left(x_{0}\right)\right\|^{2} \\
& \leq \sum_{i=1}^{N} \xi_{i}^{T}\left(A^{T} P_{1}+P_{1} A-P_{1} B B^{T} P_{1}+\kappa_{1} P_{1} P_{1}+\frac{\gamma^{2}}{\kappa_{1}} I_{n}\right) \xi_{i}+\sum_{i=1}^{N} \tilde{\eta}_{i}^{T} P_{1} B B^{T} P_{1} \tilde{\eta}_{i}
\end{aligned}
$$

where $K=B^{T} P_{1}$ and we have used the inequality $2 a^{T} b \leq \kappa a^{T} a+b^{T} b / \kappa$ for vectors $a$ and $b$ with the same dimension.

Let the Lyapunov function be $V=V_{1}+V_{2}$ with $V_{2}=\bar{V}$. By Theorem 2, two cases are considered for stability analysis, respectively.

Case 1. If the eigenvalues of the matrix $\overline{\mathcal{L}}$ are distinct, we have

$$
\begin{aligned}
\dot{V} \leq & \sum_{i=1}^{N} \xi_{i}^{T}\left(A^{T} P_{1}+P_{1} A-P_{1} B B^{T} P_{1}+\kappa_{1} P_{1} P_{1}+\frac{\gamma^{2}}{\kappa_{1}} I_{n}\right) \xi_{i} \\
& +\sum_{i=1}^{N} \tilde{\eta}_{i}^{T}\left(A^{T} P+P A-2 \bar{\alpha} C^{T} C+\kappa P P+\frac{\gamma_{0}^{2}}{\kappa} I_{n}\right) \tilde{\eta}_{i} \\
& +\sum_{i=1}^{N} \tilde{\eta}_{i}^{T} P_{1} B B^{T} P_{1} \tilde{\eta}_{i} \\
= & \sum_{i=1}^{N} \xi_{i}^{T} M_{1} \xi_{i}+\sum_{i=1}^{N} \tilde{\eta}_{i}^{T} M_{2} \tilde{\eta}_{i}
\end{aligned}
$$


where

$$
\begin{aligned}
& M_{1}=A^{T} P_{1}+P_{1} A-P_{1} B B^{T} P_{1}+\kappa_{1} P_{1} P_{1}+\frac{\gamma^{2}}{\kappa_{1}} I_{n} \\
& M_{2}=A^{T} P_{2}+P_{2} A-2 \bar{\alpha} C^{T} C+\kappa_{2} P_{2} P_{2}+\frac{\gamma_{0}^{2}}{\kappa_{2}} I_{n}+P_{1} B B^{T} P_{1}
\end{aligned}
$$

Conditions (41) and (42) guarantee $\dot{V}<0$ for $\kappa_{1}>0, \kappa_{2}>0, \gamma$ defined in (3) and $\gamma_{0}$ defined in (31), which implies $\xi_{i}(t) \rightarrow 0$ and $\tilde{\eta}_{i}(t) \rightarrow 0$ as $t \rightarrow \infty$ for all $i$.

Case 2 . If the matrix $\overline{\mathcal{L}}$ has multiple eigenvalues, we similarly have

$$
\dot{V} \leq \sum_{i=1}^{N} \xi_{i}^{T} M_{1} \xi_{i}+\sum_{i=1}^{N} \tilde{\eta}_{i}^{T} \bar{M}_{2} \tilde{\eta}_{i}
$$

where

$$
\bar{M}_{2}=A^{T} P_{2}+P_{2} A-2(\bar{\alpha}-1) C^{T} C+\kappa_{2} P_{2} P_{2}+\frac{\gamma_{0}^{2}}{\kappa_{2}} I_{n}+P_{1} B B^{T} P_{1}
$$

Conditions (43) and (44) guarantee $\dot{V}<0$, which implies $\xi_{i}(t) \rightarrow 0$ and $\tilde{\eta}_{i}(t) \rightarrow 0$ as $t \rightarrow \infty$ for all $i$.

Hence, the leader-follower consensus is achieved. This completes the proof.

Remark 5. The conditions derived in (41)-(44) can be formulated as linear matrix inequalities (LMIs) (Ding 2014). The feasibility and a possible solution can be easily checked and obtain from standard LMI routines. Note that a possible solution $P_{1}$ of (41) can be calculated first and then the possible solution $P_{2}$ of (42) for the fixed $P_{1}$ can be obtained. One can follow the same manipulation for case 2 in Theorem 3.

\section{Simulation}

In this section, an example will be employed to show some details on the consensus observerbased control designed. The system under consideration is a connection of four subsystems with a leader, where each of them described by a second order state space model as

$$
\dot{x}_{i}=\left[\begin{array}{cc}
-1 & -1 \\
1 & 0
\end{array}\right] x_{i}+\left[\begin{array}{l}
0 \\
1
\end{array}\right] u_{i}+\left[\begin{array}{c}
\sin \left(C x_{i}\right) \\
0
\end{array}\right]
$$

and

$$
C=\left[\begin{array}{ll}
1 & 0
\end{array}\right]
$$

The adjacent matrix for the connection of the followers is given by

$$
Q=\left[\begin{array}{lllll}
0 & 0 & 0 & 0 & 0 \\
1 & 0 & 1 & 0 & 0 \\
0 & 0 & 0 & 0 & 1 \\
0 & 0 & 0 & 0 & 1 \\
0 & 1 & 0 & 0 & 0
\end{array}\right]
$$


and the corresponding Laplacian matrix is obtained as

$$
\mathcal{L}=\left[\begin{array}{ccccc}
0 & 0 & 0 & 0 & 0 \\
-1 & 2 & -1 & 0 & 0 \\
0 & 0 & 1 & 0 & -1 \\
0 & 0 & 0 & 1 & -1 \\
0 & -1 & 0 & 0 & 1
\end{array}\right]
$$

The eigenvalues of $\overline{\mathcal{L}}$ are given as $\{0.2451,1,1.8774 \pm 0.7449 \mathrm{j}\}$ which are distinct. The Jordan matrix $\bar{J}$ corresponding to $\overline{\mathcal{L}}$ can be obtained as

$$
\bar{J}=\left[\begin{array}{cccc}
0.2451 & 0 & 0 & 0 \\
0 & 1 & 0 & 0 \\
0 & 0 & 1.8774 & 0.7449 \\
0 & 0 & -0.7449 & 1.8774
\end{array}\right]
$$

The nonlinear function $0.05 \sin \left(C x_{i}\right)$ in the dynamic system is globally Lipshitz. The values of the follower substates and the leader are set as $x_{1}=[0.1,-0.1]^{T}, x_{2}=[0.3,-0.3]^{T}, x_{3}=[0.2,-0.2]^{T}$, $x_{4}=[0.4,-0.4]^{T}$, and $x_{0}=[0.5,-0.5]^{T}$. Based on matrix $\overline{\mathcal{L}}$, we have $\alpha=0.2451$. From (48), we have

$$
\phi\left(x_{i}\right)=\left[\begin{array}{c}
\sin \left(C x_{i}\right) \\
0
\end{array}\right]
$$

The Lipschitz constant is $\gamma=0.05$ and the bound in Lemmas 2 and 3 can be calculated as $\gamma_{0}=0.0115$. With $\kappa_{1}=1.0$, and $\kappa_{2}=1.0$, the control gain and the observer gain can be computed respectively as

$$
\begin{gathered}
K=[-0.01890 .2759] \\
L=\left[\begin{array}{l}
0.9708 \\
0.1058
\end{array}\right]
\end{gathered}
$$

where

$$
\begin{aligned}
& P_{1}=\left[\begin{array}{cc}
0.2763 & -0.0189 \\
-0.0189 & 0.2759
\end{array}\right] \\
& P_{2}=\left[\begin{array}{cc}
1.0424 & -0.1132 \\
-0.1132 & 1.0382
\end{array}\right]
\end{aligned}
$$

Figures 1 and 2 show, respectively, the time evolutions of the sub-state 1 and 2 of the leader and all followers subject to the controller (37) with $\gamma=0.05$. The estimation errors of all sub-states of each follower are presented in Figures 3 and 4, which demonstrate that the proposed consensus observer recovers the state of each follower. Since the conditions (41)-(42) derived in Theorem 3 are sufficient conditions for the control gain design of the multi-agent systems with nonlinearities, these conditions may be conservative for a given Lipschitz nonlinear function in the control gain design. As shown in Figures 5 and 6, the same control gain can also achieve the leader-follower consensus for the nonlinearities with a larger Lipschitz constant $\gamma=0.8$. Noticeably, it took a bit longer time to achieve the consensus tracking due to a stronger nonlinearity. In addition, the 


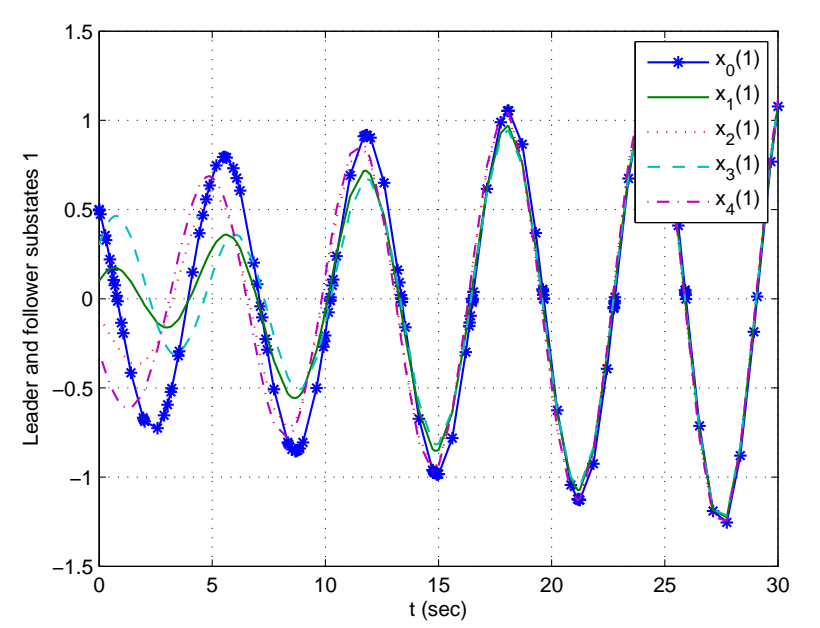

Figure 1. Time evolution of sub-state 1 of the leader and each follower: $\gamma=0.05$.

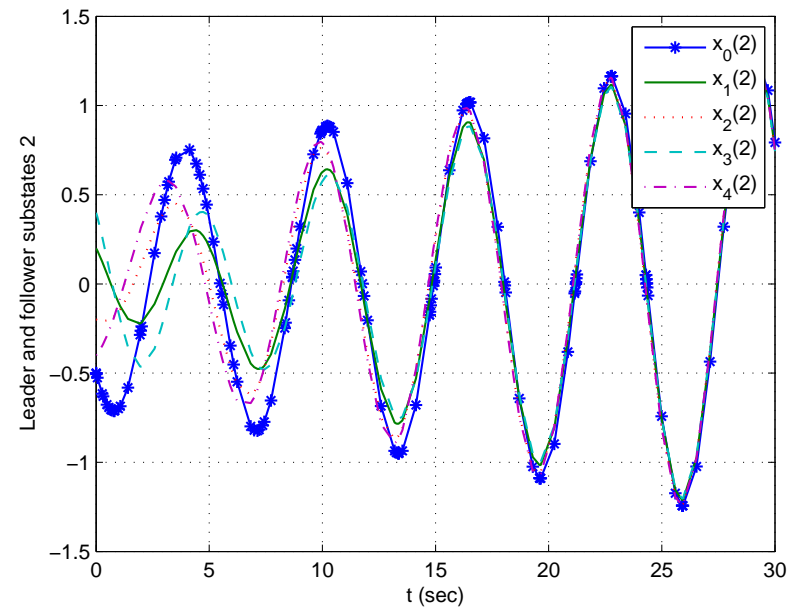

Figure 2. Time evolution of sub-state 2 of the leader and each follower: $\gamma=0.05$.

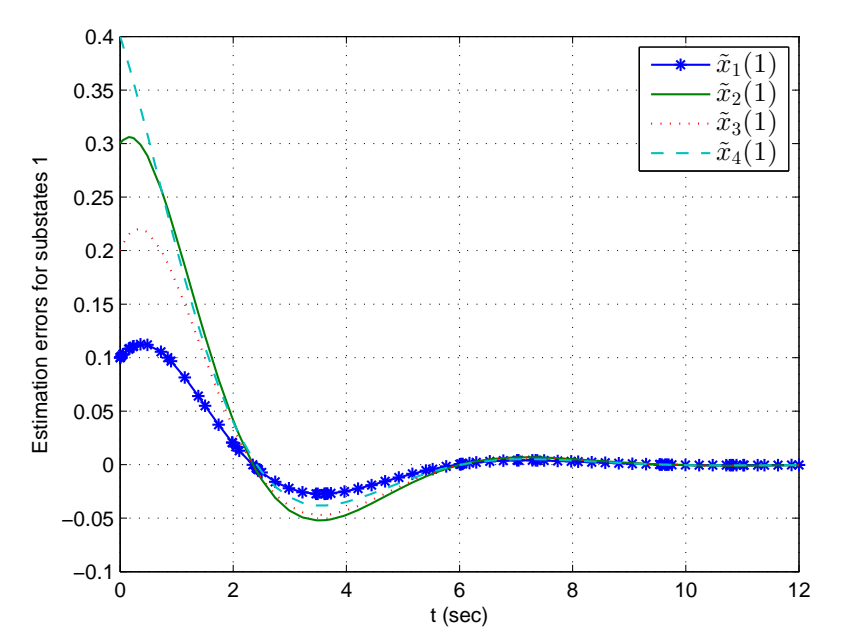

Figure 3. Estimation error for sub-state 1 of each follower: $\gamma=0.05$.

. Time evolution of sub-state 1 of the leader and each follower: $\gamma=0.05$. 


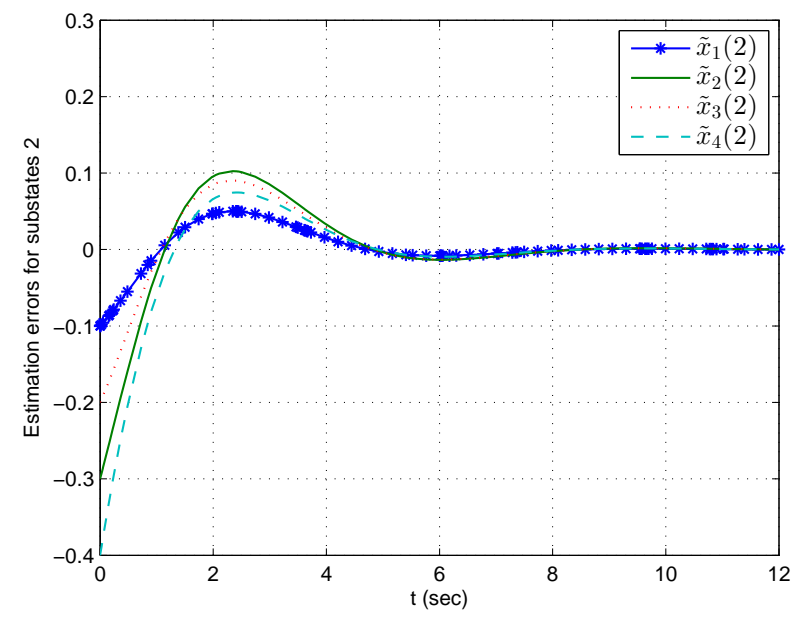

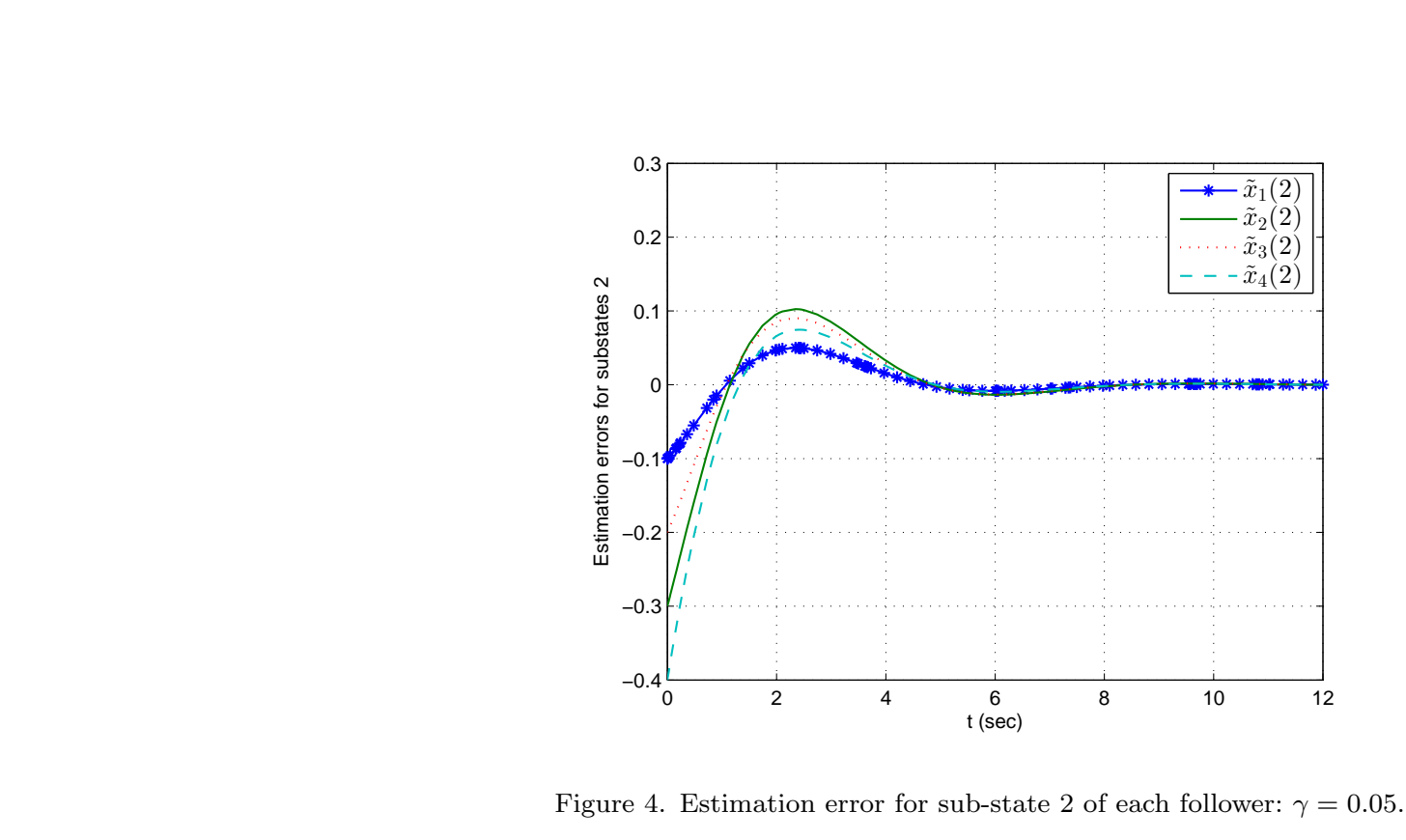

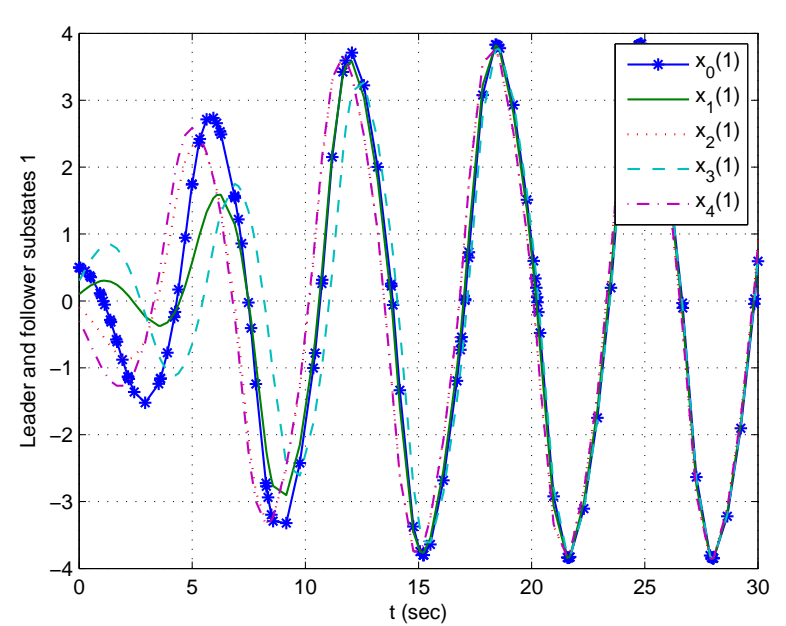

Figure 5. Time evolution of sub-state 1 of the leader and each follower: $\gamma=0.8$.

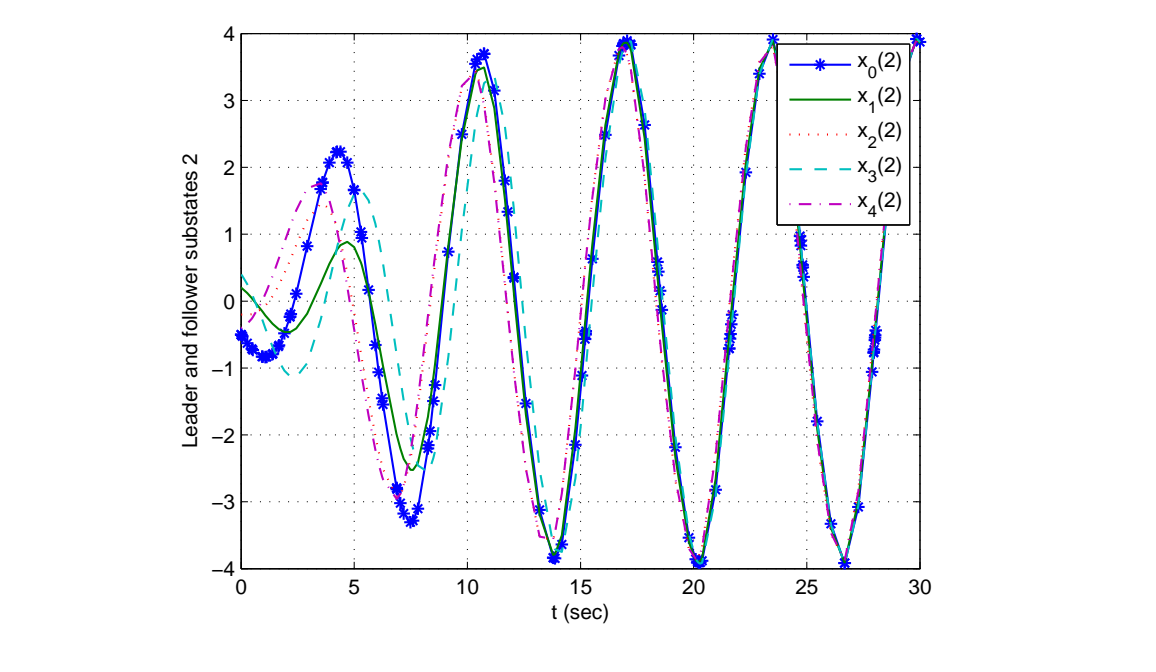

Figure 6. Time evolution of sub-state 1 of the leader and each follower: $\gamma=0.8$.

Figure 6. Time evolution of sub-state 1 of the leader and each follower: $\gamma=0.8$.

Figue 5. The evelution of sh-state 1 of the leader and each follow: $\gamma=0.8$. 
subsystem trajectories shown in Figures 1 and 2 are different from those in Figures 5 and 6 due to the difference in the nonlinear term.

\section{Conclusion}

In this paper, both state and output feedback leader-follower consensus controllers have been proposed for the multi-agent systems with Lipschitz nonlinearity in networks subject to a spanning tree topology. The global stability is guaranteed by a careful study of the Laplacian structure and some sufficient conditions, if the nonlinear terms are globally Lipschitz. This study is also applicable to consensus control of local Lipschitz nonlinear systems around operating points with the consideration of stability in a small neighbourhood, since the Lipschitz constants are getting smaller as it approaches the operating points. This means that the results obtained in this paper can be used to check the operating region, in which the stability can be ensured by checking the local Lipschitz constants.

\section{Acknowledgement}

The first author would like to thank University Teknikal Malaysia Melaka (UTeM) for their sponsorship. The second author would like to thank the support of the National Natural Science Foundation of China (No.61203022) and the China Scholarship Council (CSC).

\section{References}

Aboky, C., Sallet, G., Vivalda, J. C. (2002), 'Observers for lipschitz non-linear systems', International Journal of Control, 75, 204-212.

Cai, H., Huang, J. (2014), 'The leader following consensus control of multiple rigid spacecraft systems', Automatica, 50, 1109-1115.

Cao, Y., Ren, W. (2010), 'Multi-agent consensus using both current and outdated states with fixed and undirected interaction', Journal of Intelligent Robotics Systems,58, 95-106.

Chen, F., Chen, Z., Xiang, L., Liu, Z., Yuan, Z. (2009), 'Reaching a consensus via pinning control', Automatica, 45, 1215-1220.

Chen, G., Lewis, F. L., Xie, L. (2011), 'Finite-time distributed consensus via binary control protocols', Automatica, 47, 1962-1968.

Cheng, L., Hou, Z., Tan, M., Lin, Y., Zhang, W. (2010), 'Neural-network-based adaptive leader-following control for multi-agent systems with uncertainties', Transaction of Neural Network, 21, 1351-1358.

Cui, Y., Jia, Y. (2012), 'Robust $L_{2}-L_{\infty}$ consensus control for uncertain high-order multi-agent systems with time-delay', International Journal of Systems Science, 45, 427-438.

Ding, Z. (2013), 'Consensus output regulation of a class of heterogeneous nonlinear systems', IEEE Transactions on Automatic Control, 58, 2648-2653.

Ding, Z. (2013), 'Nonlinear and adaptive control systems', London:IET.

Ding, Z. (2014), 'Consensus control of a class of Lipschitz nonlinear systems', International Journal of Control, 87, 2372-2382.

Dong, Y., Huang, J. (2014), 'Cooperative global output regulation for a class of nonlinear multi-agent systems', IEEE Transactions on Automatic Control, 59, 1348-1354.

Fax, J., Murray, R. (2004), "Information flow and cooperative control of vehicle formations.", IEEE Transactions on Automatic Control, 49, 1465-1476.

Godsil, C., Royle, G. (2001), 'Algebraic Graph Theory', Springer.

He, W., Cao, J. (2011), 'Consensus Control for high-order multi-agent systems', IET Control Theory Applications, 5, 231-238.

Hong, Y., Hu, J., Gao, L. (2006), 'Tracking control for multi-agent consensus with an active leader and variable topology', Automatica, 46, 1177-1182. 
Hong, Y., Wang, X., Jiang, Z. (2010), 'Distributed output regulation of leader-follower multi-agent systems', International Journal of Robust and Nonlinear Control, 23, 48-66.

Horn, R.A, Johnson, C.R. (1990), 'Matrix Analysis', Cambridge University Press.

Li, Z., Duan, Z., Huang, L. (2009), 'Leader-follower consensus of multi-agent systems', American Control Conference, 3256-3261.

Li, Z., Duan, Z., Chen, G., Huang, L. (2010), 'Consensus of multi-agent systems and synchronization of complex networks: A unified viewpoint', IEEE Transactions on Circuits and Systems I: Regular Papers, $57,213-224$.

Li, Z., Ren, W., Liu, X., Fu, M. (2012), 'Global $H_{\infty}$ consensus of multi-agent systems with lipschitz nonlinear dynamics', IET Control Theory Applications, 6, 2041-2048.

Li, Z., Ren, W., Liu, X., Fu, M.(2013), 'Consensus of multi-agent systems with general linear and lipschitz dynamics using distributed adaptive protocols', IEEE Transactions on Automatic Control, 58, 17861791.

Liu, S., Xie, L., Zhang, H.(2011), 'Distributed consensus for multi-agent systems with delays and noises in transmission channels', Automatica, 47, 920-934.

Liu, Y., Jia, Y. (2012), ' $H_{\infty}$ consensus control for multi-agent systems with linear coupling dynamics and communication delays', International Journal of System Science, 43, 50-62.

Olfati-Saber, R., Murray, R. (2004), 'Consensus problems in networks of agents with switching topology and time-delays', IEEE Transactions on Automatic Control, 49, 1520-1533.

Olfati-Saber, R., Murray, R. (2003), 'Agreement problems in networks with directed graphs and switching topology', Proceeding. 42nd IEEE Conference on Decision and Control, 4, 4126-4132.

Phanomchoeng, G., Rajamani, R. (2010), 'Observer design for lipschitz nonlinear systems using Riccati equations', American Control Conference, 6060-6065.

Ren, W. (2007), 'Multi-vehicle consensus with a time-varying reference state', System and Control Letters, $56,474-483$.

Ren, W. (2007), 'Consensus strategies for cooperative control of vehicle formations', IET Control Theory Applications, 1, 505-512.

Ren, W., Atkins, E. (2007), 'Distributed multi-vehicle coordinated control via local information exchange', International Journal of Robust and Nonlinear Control, 17, 1002-1033.

Ren, W., Moore, K. L., Chen, Y. (2007), 'High-order and model reference consensus algorithms in cooperative control of multi-vehicle systems', Journal of Dynamic Systems, Measurement, and Control, 129, 678-688.

Rajamani, R. (1998), 'Observers for lipschitz nonlinear systems', IEEE Transactions on Automatic Control, 43, 397-401.

Rajamani, R., Cho, Y. M. (2002), 'Existence and design of observers for nonlinear systems: Relation to distance to unobservability', International Journal of Control, 75, 717-731.

Song, Q., Cao, J., Yu, W. (2010), 'Second-order leader-following consensus of nonlinear multi-agent systems via pinning control', Systems and Control Letters, 59, 553-562.

Su, H., Chen, M.Z.Q., Lam, J., Lin, Z. (2010), 'Semi-global leader-following consensus of linear multi-agent systems with input saturation via low gain feedback', IEEE Transactions on Circuits and Systems, 60, 1881-1889.

Ni, W., Cheng, D. (2010), 'Leader-following consensus of multi-agent systems under fixed and switching topologies', Systems and Control Letters, 59, 209-217.

Wang, y., Cheng, L., Hou, Z., Tan, M., Wang, M. (2014), 'Containment control of multi-agent systems in a noisy communication environment', Automatica, 50, 1922-1928.

Zhang, H., Lewis, F.L., Das, A. (2011), 'Optimal design for synchronization of cooperative systems: State Feedback, observer and output feedbacks', IEEE Transactions on Automatic Control, 56, 1948-1952.

Zuo, Z., Tie, L.(2014), 'Distributed robust finite-time nonlinear consensus protocols for multi-agent systems', International Journal of Systems Science, Published online. 